\title{
High-dose dexamethasone induced LPS-stimulated rat alveolar macrophages apoptosis
}

This article was published in the following Dove Press journal:

Drug Design, Development and Therapy

25 October 2017

Number of times this article has been viewed

\author{
Si Zeng ${ }^{1, *}$ \\ Hui Qiao $2, *$ \\ Xue-wen Lv' \\ Dan Fan' \\ Tong Liu' \\ Dongli Xie' \\ 'Department of Anesthesiology, \\ Sichuan Academy of Medical \\ Sciences \& Sichuan Provincial \\ People's Hospital, Chengdu, \\ ${ }^{2}$ Department of Anesthesiology, \\ The Eye and ENT Hospital of \\ Fudan University, Shanghai, China \\ *These authors contributed equally \\ to this work
}

\begin{abstract}
Prolonged administration of an excessive dose of corticosteroids proved to be harmful for patients with acute lung injury (ALI). A previous study has found that repeated administration of an excessive dose of methylprednisolone reduced alveolar macrophages (AMs) in bronchoalveolar lavage fluid (BALF) with an unknown mechanism. This study aimed to investigate the effect of excessive use of dexamethasone (Dex) on BALF AMs in vitro. Transmission electron microscopy and DNA fragmentation analysis demonstrated that $10^{-4}$ and $10^{-5} \mathrm{M}$ Dex induced lipopolysaccharide-stimulated rat AMs apoptosis with downregulation of tumor necrosis factor- $\alpha$, interleukin (IL)-12 and upregulation of IL-10, transforming growth factor- $\beta$. These results indicated that apoptosis might be a novel contribution involved in the detrimental effect of excessive dose of Dex clinically used to treat ALI.
\end{abstract}

Keywords: dexamethasone, alveolar macrophage, lipopolysaccharide, acute lung injury, apoptosis, inflammatory cytokines

\section{Introduction}

Acute lung injury (ALI) and adult respiratory distress syndrome (ARDS) are devastating clinical syndromes that are characterized by hypoxemia and increased breathing effort resulting from gas exchange impairment with mortality at a high level of $43 \% .{ }^{1}$ Glucocorticosteroids (GCs) are important drugs to treat ALI/ARDS due to their anti-inflammatory and anti-fibrotic effects. However, numerous trials have reported that excessive corticosteroid administration for ARDS resulted in no benefit in survival, and was even detrimental. ${ }^{2-4}$ The specific role of GCs in ALI/ARDS and how to use GCs to treat ALI/ARDS remain controversial.

Alveolar macrophages (AMs) are predominant immune cells residing in the pulmonary alveolus and are responsible for inflammatory responses activation which is necessary to eliminate pathogens. ${ }^{5}$ AMs play a vital role not only in lung inflammation and ALI but also in the process of the resolution of inflammation. ${ }^{6}$ In our previous study, we found that repeated administration of an excessive dose of methylprednisolone consecutively reduced AMs in rat bronchoalveolar lavage fluid (BALF). ${ }^{7}$ In this study, we explored the effects of high-dose dexamethasone (Dex) on AMs in vitro and analyzed the underlying mechanism regarding apoptosis and dysregulated expression of cytokine.

\section{Materials and methods Isolation and culture of rat AMs}

The animal protocol was approved by the ethical committee of Sichuan Academy of Medical Sciences, Sichuan Provincial People's Hospital, Chengdu, China and carried 
out within the guidelines of the Animal Care Committee. Adult male Sprague Dawley rats (200-250 g) were used for isolation of AMs. The rats were anesthetized by intraperitoneal application of $100 \mathrm{mg} \cdot \mathrm{kg}^{-1}$ sodium pentobarbital (Bioz, Palo Alto, CA, USA) followed by blood collection from the abdominal aorta. After median thoracotomy, the lung was lavaged 10 times with $5 \mathrm{~mL}$ ice-cold phosphatebuffered saline (PBS; Thermo Fisher Scientific, Waltham, MA, USA) solution by cannulating the trachea with a shortened 14-gauge needle attached to a 5-mL syringe, which was allowed to passively run out after each instillation while gently massaging the lung. AMs and cell-free lavage were obtained by centrifugation of BALF at $300 \times g$ for 10 minutes at $4{ }^{\circ} \mathrm{C}$. Cells were washed three times in $\mathrm{Ca}^{2+} / \mathrm{Mg}^{2+}$-free PBS and were finally suspended in low glucose Dulbecco's Modified Eagle's Medium (GE Healthcare Life Sciences, Chicago, IL, USA) containing 10\% fetal bovine serum (endotoxin $<10.0 \mathrm{EU} \cdot \mathrm{mL}^{-1}$ ), $100 \mathrm{U} \cdot \mathrm{mL}^{-1}$ of penicillin, and $100 \mu \mathrm{g} \cdot \mathrm{mL}^{-1}$ of streptomycin. The cells were cultured in 6 -well culture plates $\left(10^{6}\right.$ cells/well) or 96 -well culture plates ( $10^{5}$ cells/well). After incubation for 2 hours at $37^{\circ} \mathrm{C}$ in a $5 \%$ $\mathrm{CO}_{2}$ humidified atmosphere, the non-adherent cells were removed by washing twice with PBS. AMs were incubated overnight to make them quiescent and ready for further experimental steps. Cell viability and purity were determined by trypan blue exclusion and Wright-Giemsa dye assay with the threshold set no less than $95 \%$ and $98 \%$, respectively.

\section{Study design}

After incubating for 24 hours, AMs were randomly divided into six groups: 1) control group, AMs were incubated with PBS; 2) lipopolysaccharide (LPS; Sigma-Aldrich, St. Louis, MO, USA) group, AMs were stimulated with LPS (1 $\mu \mathrm{g} / \mathrm{mL})$; 3) $\mathrm{LPS}+10^{-4} \mathrm{M}$ Dex group (Sigma-Aldrich); 4) LPS $+10^{-5}$ M Dex group; 5) LPS $+10^{-6} \mathrm{M}$ Dex group; 6) $\mathrm{LPS}+10^{-7} \mathrm{M}$ Dex group; AMs were incubated with LPS for 24 hours and then stimulated with different doses of Dex $\left(10^{-4}, 10^{-5}, 10^{-6}\right.$, or $10^{-7} \mathrm{M}$ ) for another 6,24 , or 48 hours. AMs were then cultured for assay-specific periods of time in a humidified chamber containing $5 \% \mathrm{CO}_{2}$ at $37^{\circ} \mathrm{C}$. Cell-free supernatants were kept at $-80^{\circ} \mathrm{C}$ for later analysis.

\section{Reagents}

LPS (Escherichia coli, serotype 055:B5), Dex (D8893), 3-(4,5-dimethyl-2-thiazolyl)-2,5-diphenyl-2H-tetrazolium bromide (MTT), and trypan blue dye were obtained from Sigma-Aldrich. Cell culture reagents were purchased from GE Healthcare Life Sciences. Rat interleukin (IL)-10 and tumor necrosis factor (TNF)- $\alpha$ enzyme-linked immunosorbent assay (ELISA) kits were prepared from USCN Life Science, Wuhan, China. IL-12 and transforming growth factor (TGF)- $\beta$ ELISA kits were purchased from CUSABIO Life Science, College Park, MD, USA.

\section{Cell viability assay}

MTT assay was used to assess cell viability. MTT $(5 \mathrm{mg} / \mathrm{mL})$ was added to the replaced culture medium in 96-well plates $\left(10^{5}\right.$ cells/well) after being treated with indicated concentrations of PBS, LPS, or LPS + Dex $\left(10^{-4}, 10^{-5}, 10^{-6}\right.$, and $\left.10^{-7} \mathrm{M}\right)$ for 6,24 , and 48 hours. After incubation for another 4 hours at $37^{\circ} \mathrm{C}$, the culture medium was removed and formazan formed by viable cells was solubilized in $100 \mu \mathrm{L}$ dimethyl sulfoxide. The optical density was measured using a microplate reader (BioTek Instruments, Winooski, VT, USA) at $570 \mathrm{~nm}$.

\section{Transmission electron microscopy (TEM)}

AM samples were treated as indicated groups, harvested, and fixed in $2.5 \%$ glutaraldehyde in $0.1 \mathrm{M}$ sodium cacodylate buffer ( $\mathrm{pH} 7.3$ ). Additional postfixation in $1 \%$ osmium tetroxide was followed by dehydration through an ascending concentration series of ethanol before embedding in resin (LR White). Ultrathin (100 nm) sections were cut with an LKB ultramicrotome (LKB-NOVA, Bromma, Sweden), equipped on copper grids (300-mesh), counterstained with lead citrate, and examined under a JEOL JEM 1230 electron microscope (JEOL, Tokyo, Japan). Morphometry analyses were performed for each sample from four random grid squares covering an area of $0.03 \mathrm{~mm}^{2}$.

\section{DNA laddering detection}

DNA fragmentation in apoptotic cells was detected by agarose gel electrophoresis. In brief, AMs treated with LPS and Dex were pelleted $(200 \times g, 5$ minutes $)$ and lysed in detergent buffer (20 mM Tris, $0.4 \mathrm{mM}$ EDTA, and $0.4 \%$ Triton-X) for $15 \mathrm{~min}$ utes on ice $\left(4^{\circ} \mathrm{C}\right)$. After centrifugation $(13,000 \times g, 5$ minutes, $4^{\circ} \mathrm{C}$ ), the supernatants were collected in a new microfuge tube. DNA solution was precipitated overnight with an equal volume of room temperature isopropanol after adjustment of the salt concentration. The DNA pellet was washed twice by adding $70 \%$ ethanol, air-dried, and redissolved in Tris-EDTA buffer. The extracted DNA was separated electrophoretically on a $1.5 \%$ agarose gel containing $0.5 \mu \mathrm{g} / \mathrm{mL}$ ethidium bromide and visualized by ultraviolet transillumination.

\section{Determination of inflammatory cytokines}

The concentration of TNF- $\alpha$, IL-12, IL-10, and TGF- $\beta$ in cell growth medium supernatant was detected by ELISA 
kits (USCN, CUSABIO) according to the manufacturers' instructions.

\section{Statistical analysis}

Statistical analysis was performed using SPSS software version 19.0 (IBM Corporation, Armonk, NY, USA). All the parametric values were expressed as means \pm standard deviation. Data were analyzed using one-way analysis of variance. Results with a value of $P<0.05$ were considered to be statistically significant, based on two-tailed analysis.

\section{Results}

\section{Effect of Dex on AMs morphology}

Both fibroblastoid and round cells could be found in normal AMs (Figure 1A). LPS treatment caused AMs to adopt a fully differentiated amoeboid morphology (Figure 1B). Dextreated AMs showed a condensed morphology (Figure 1C-F) and decreased survival time.

\section{Cell viability assay}

LPS treatment for 6 hours significantly promoted cell viability as determined by the MTT assay when compared with 24 and 48 hours $(P<0.01)$. Co-treatment of Dex decreased cell viability in LPS-treated AMs, most robustly at 24 hours $(P<0.05$, Figure 2$)$.

\section{TEM}

Normal AMs presented an integrated nuclear membrane, relatively homogeneous chromatin, and extensive interdigitations of membrane and microvilli (Figure 3A). Devour vesicles could be detected in LPS-treated AMs, indicating active phagocytosis (Figure 3B). Co-treatment with Dex $10^{-5} \mathrm{M}$ resulted in nuclear chromatin concentration and edge accumulation (Figure 3C), while apoptosis bodies and various vacuoles emerged in the cytoplasm and membrane microvilli disappeared when co-treated with $10^{-4} \mathrm{M}$ Dex (Figure 3D).

\section{DNA fragmentation analysis}

DNA fragmentation, one of the archetypal biochemical characteristics of apoptosis, was determined to further explicate the apoptotic induction by high-dose Dex in AMs. As shown in Figure 4, distinct DNA ladders appeared in both Dex $10^{-4} \mathrm{M}(\mathrm{C})$ and Dex $10^{-5} \mathrm{M}(\mathrm{D})$ group.

\section{TNF- $\alpha$, IL- I2, IL- I 0, and TGF- $\beta$ assays in cell culture medium supernatant}

As shown in Figure 5, the production of TNF- $\alpha$ in the Dex group was significantly lesser than that in the LPS group $(P<0.01)$. Treatment with Dex for 24 and 48 hours also resulted in decreased IL-12 production compared with the LPS group $(P<0.05$, Figure 6). IL-10 concentration was higher in Dex group than in the PBS group $(P<0.05$, Figure 7). The production of TGF- $\beta$ in LPS $+10^{-4} \mathrm{M}$ Dex group significantly increased when compared with the LPS group $(P<0.05$, Figure 8$)$.

\section{Discussion}

In our previous research, repeated administration of high dose of methylprednisolone resulted in decreased number
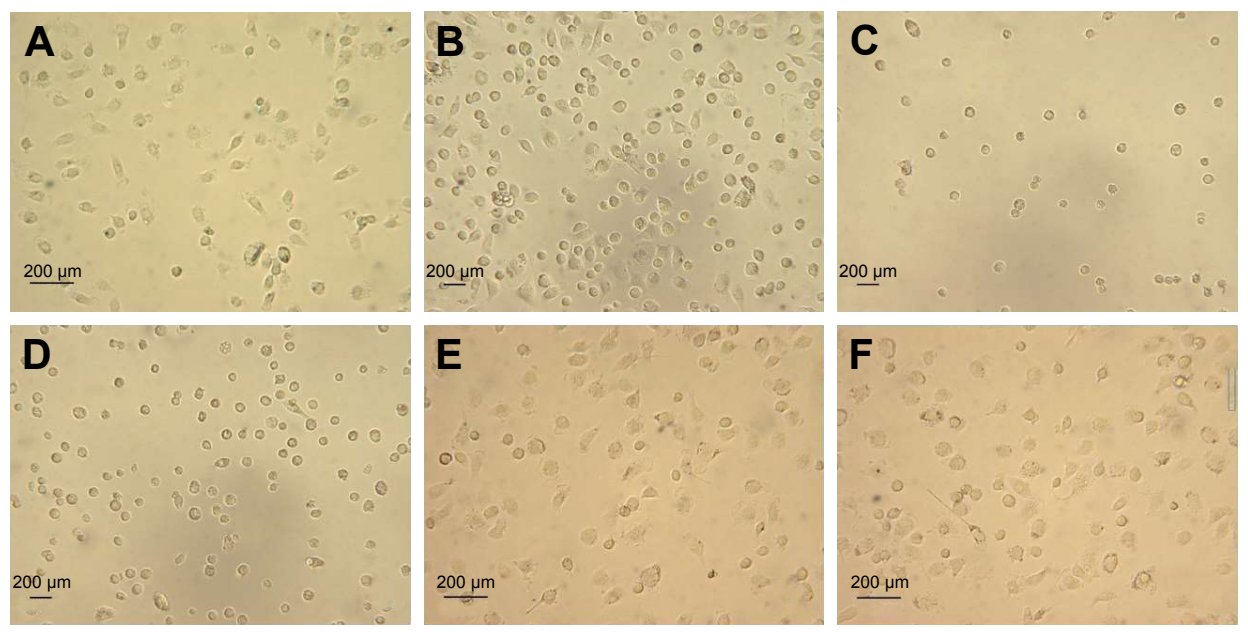

Figure I Morphological changes of rat AM.

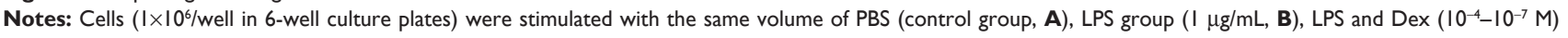
(C-F), respectively, for 48 hours. Original magnification $\times 200$.

Abbreviations: AM, alveolar macrophage; PBS, phosphate-buffered saline; LPS, lipopolysaccharide; Dex, dexamethasone. 


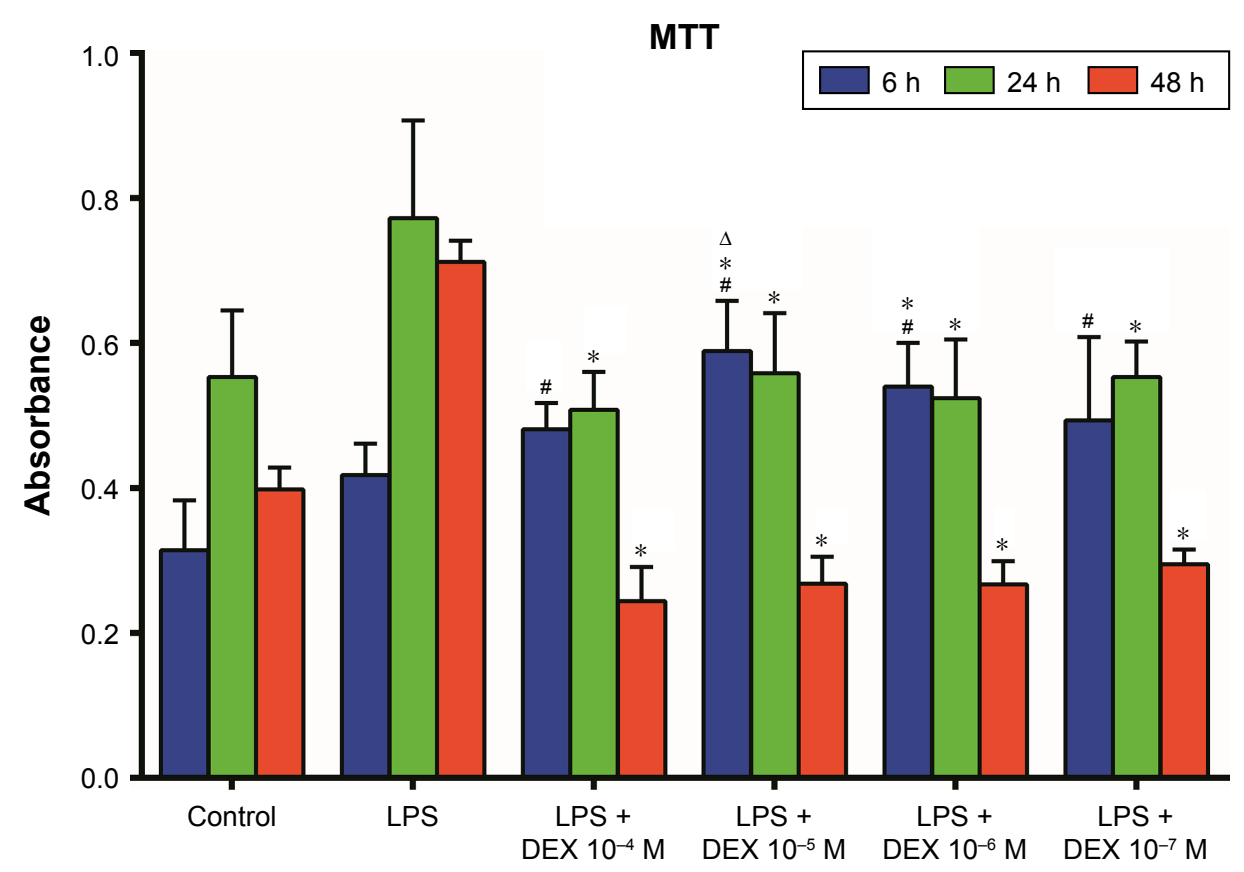

Figure 2 Effect of Dex on cell viability.

Notes: Cells ( $1 \times 10^{5} /$ well in 96-well culture plates) were stimulated with the same volume of PBS (control group), LPS (I $\left.\mu \mathrm{gg} / \mathrm{mL}^{2}\right), \mathrm{LPS}$ and Dex (I0-4-I0-7 M), respectively, for 6,24 , and 48 hours. Cell viability was assessed by the MTT assay, as described in the "Materials and methods" section. Data were expressed as mean \pm SD ( $=5$ ). $* P<0.05$ versus LPS. ${ }^{*} P<0.05$ versus PBS. ${ }^{\Delta P}<0.05$ versus $L P S+D e x 10^{-4} \mathrm{M}$.

Abbreviations: h, hours; PBS, phosphate-buffered saline; LPS, lipopolysaccharide; Dex, dexamethasone; SD, standard deviation; MTT, 3-(4,5-dimethyl-2-thiazolyl)-2,5diphenyl-2H-tetrazolium bromide.

of AMs in rat lung tissue. In this study, high-dose Dex $\left(10^{-4}\right.$ and $\left.10^{-5} \mathrm{M}\right)$ administration turned out to induce apoptosis of LPS-stimulated rat AMs and increase the production of TGF- $\beta$. This result might help explain the detrimental effect of prolonged high-dose methylprednisolone used to treat ALI.

Given that AMs act as a defender for the alveolarblood interface against respiratory organisms, the role of AMs in the pathogenesis of ALI/ARDS has been extensively investigated. ${ }^{6}$ It is widely accepted that AMs serve as dominant phagocytes responsible for elimination of infectious, toxic, or allergenic particles from airways. ${ }^{8}$ During tissue repair, activated macrophages serve the function of antiinflammation and potential "wound healing"; therefore, AMs have the capability to be conducive to tissue healing in many ways. ${ }^{9,10}$ In the current study, we found that a relatively high dose of Dex could induce apoptosis of AMs in vitro. We proposed that decreased AMs could weaken the ability of selfrepair in lung tissue. Meanwhile, apoptosis of AMs caused by Dex treatment may impair the pulmonary immune capacity. As a consequence, patients treated with high-dose Dex are at a significantly increased risk of secondary infections. ${ }^{11,12}$

Apoptosis is programmed cell death that regulates the number and fate of macrophages ${ }^{13} \mathrm{GCs}$ affect various functions of immune cells such as cellular motility, adhesion, phagocytosis, chemotaxis, and reactive oxygen metabolism. ${ }^{14}$ GCs also increased apoptosis in various polymorphonuclear cells, for example, eosinophils, T lymphocytes, and fibroblasts. ${ }^{15-17}$ Our current study found that Dex $\left(10^{-4}\right.$ and $10^{-5} \mathrm{M}$ ) could induce apoptosis of LPS-stimulated rat AMs. The possible mechanism may be that modulation of the expression of glucocorticoid receptor (GR), as dominantnegative GR $\beta$, as well as the glucocorticoid-induced leucine zipper mRNA, increased while GR $\alpha$ decreased when preincubated with LPS. ${ }^{18}$

Proinflammatory cytokines play a vital role in initiation and propagation of the inflammation of ALI/ARDS. ${ }^{19} \mathrm{TNF}-\alpha$ is the most widely studied pleiotropic cytokine of the TNF superfamily. Lungs from TNF- $\alpha$-deficient mice were less likely to be injured compared with those from wild-type mice. ${ }^{20}$ Our results indicated that Dex reduced TNF- $\alpha$ production in LPS-stimulated AMs. These effects were mediated by transcription factors, such as NF- $\mathrm{KB}$ and downstream pathways of synthesis of inflammatory mediators through the regulation of glucocorticoid-GR. ${ }^{21}$

IL-12 is the main stimulator of IFN- $\gamma$ production and of the development of mitogenic response of T-cells. IL-12 suppressed allergic lung inflammation largely through IFN- $\gamma$ production and an IL-10-dependent mechanism. ${ }^{22}$ Activation of the toll-like receptors-4 pathways is known to upregulate 

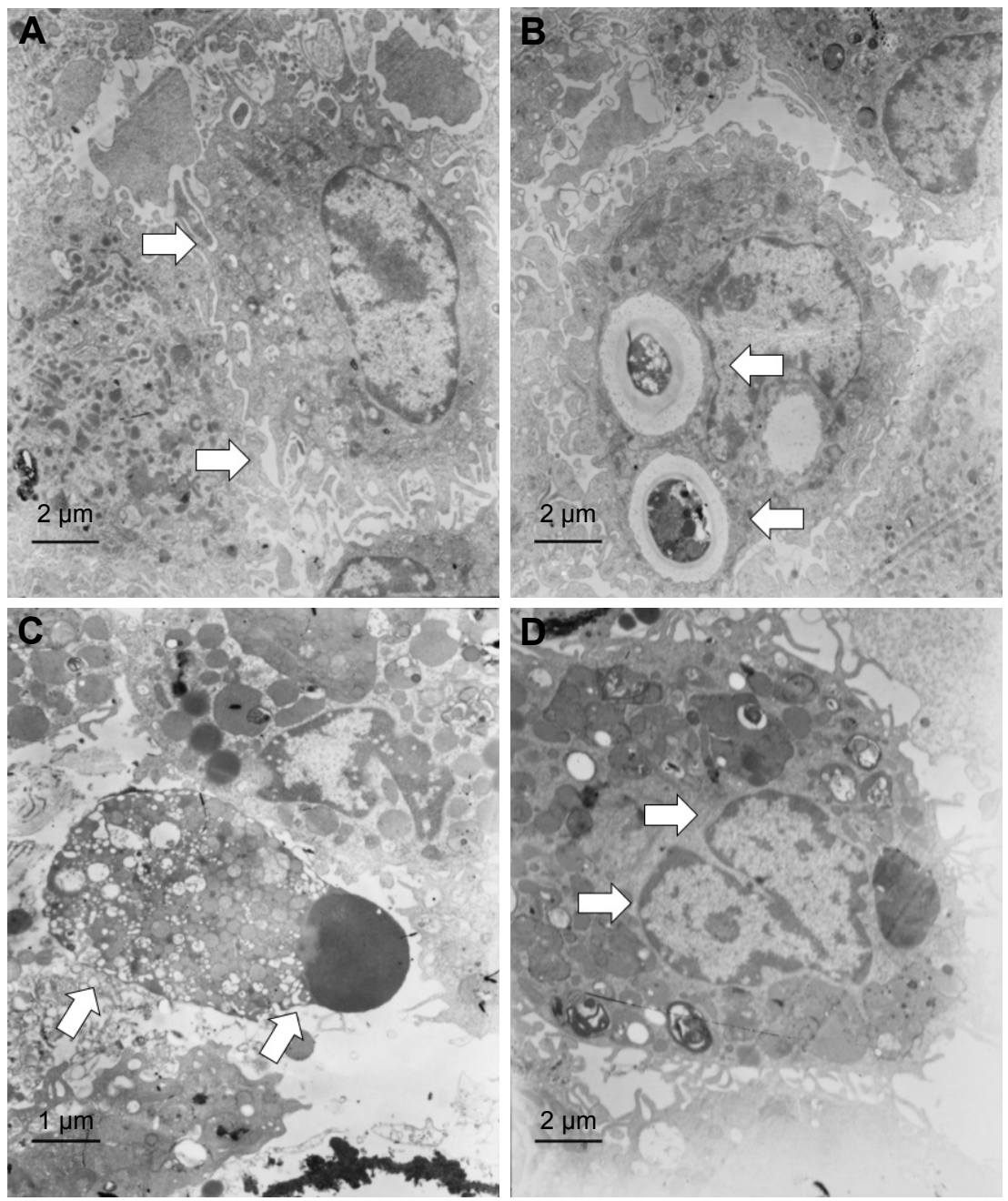

Figure 3 Transmission electron microscopy.

Notes: (A) Cells in the control group showed integrated nuclear membrane, relatively homogeneous chromatin, and extensive membrane interdigitations and microvilli (arrows). Magnification $\times 5,000$. (B) Devour vesicles (arrows) in the LPS group. Magnification $\times 5,000$. (C) Apoptosis bodies (arrows) in the LPS + Dex $10^{-4}$ M group. Magnification $\times 5,000$. (D) In the LPS + Dex 10-5 M group, stimulation of Dex induced nuclear chromatin concentration and edge accumulation (arrows). Magnification $\times 10,000$. Abbreviations: LPS, lipopolysaccharide; Dex, dexamethasone.

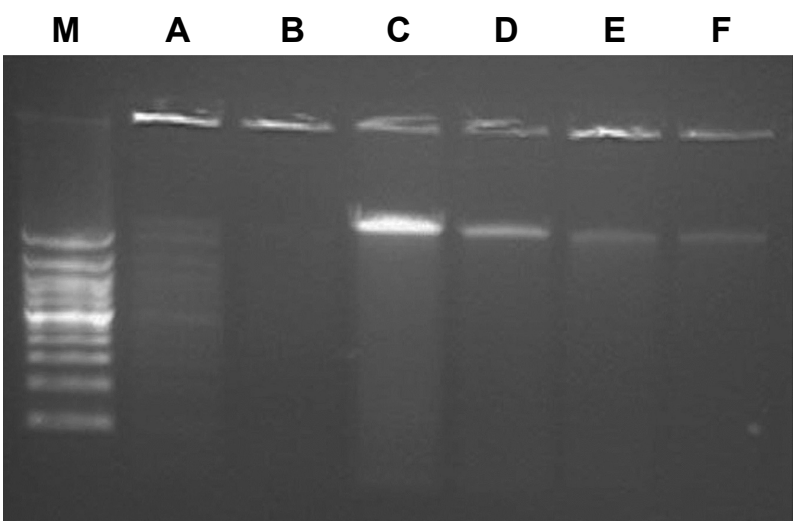

Figure 4 The apoptotic DNA ladder induced by different doses of Dex

Notes: Cells $\left(2 \times 10^{6} /\right.$ well in 6-well culture plates) were stimulated with the same volume of PBS (control group, A), LPS (I $\mu \mathrm{g} / \mathrm{mL}$, B), LPS and Dex $\left(10^{-4}-10^{-7} \mathrm{M}\right)$ (C-F), respectively, for 48 hours. Then, DNA was extracted and electrophoresed on agarose gel.

Abbreviations: PBS, phosphate-buffered saline; LPS, lipopolysaccharide; Dex, dexamethasone.
IL-12 production which could enhance the production of IFN- $\gamma$ and IL- $1 \beta$ to promote antibody-induced joint inflammation. ${ }^{23}$ Consistent with previous studies, Dex was found to reduce IL-12 production in LPS-stimulated AMs.

In this study, Dex could increase the IL-10 level in AMs. IL-10 is known as a potent anti-inflammatory cytokine, which reduces the production of proinflammatory cytokines in activated macrophages. ${ }^{24}$ IL-10-deficient mice failed to accelerate lung injury resolution. ${ }^{25}$ Dex also induced the production of IL-10 in monocyte-derived dendritic cells. ${ }^{26}$ Visser et al found that high concentrations of GC receptor antagonist (RU486) inhibited generation of IL-10, indicating that GCs may exert a stimulatory effect on the production of IL-10. ${ }^{27}$

TGF- $\beta$ is a multifunctional cytokine that facilitates the regulation of cell differentiation, morphogenesis, angiogenesis, 


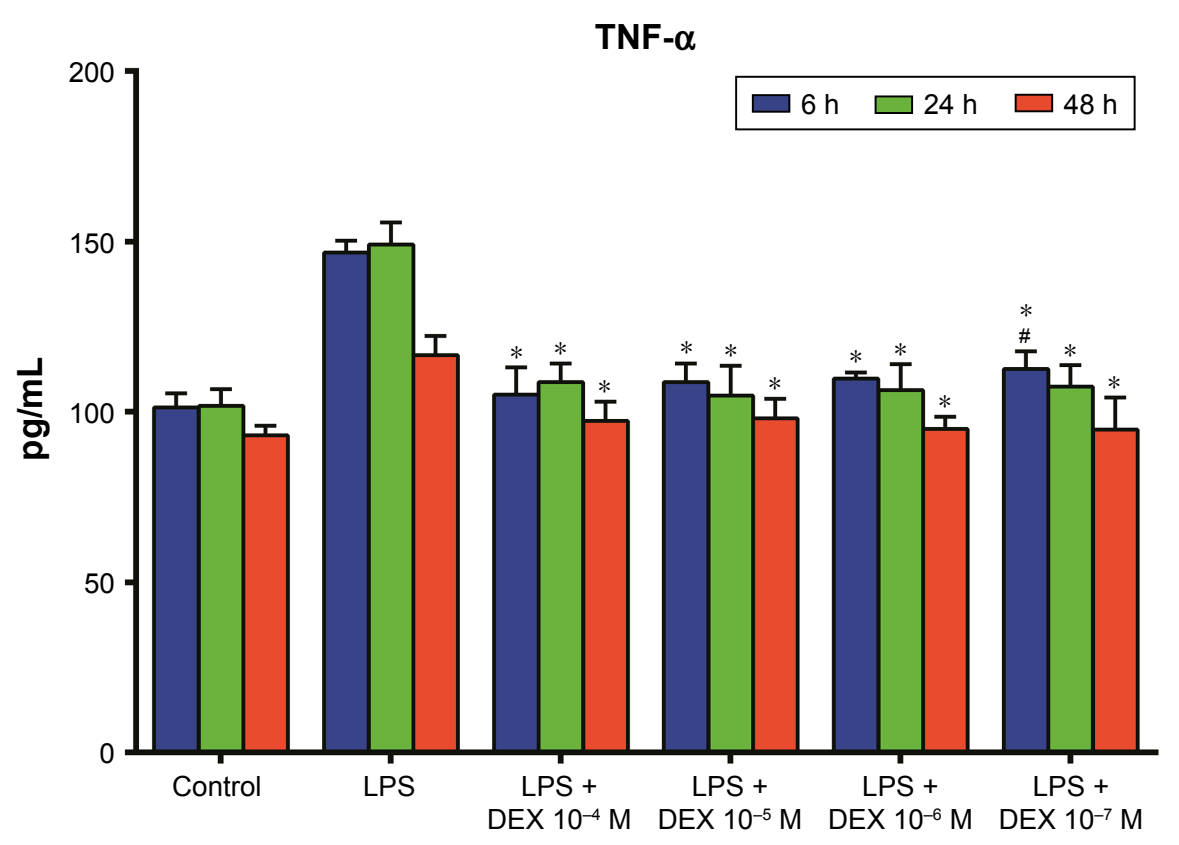

Figure 5 Effect of Dex on TNF- $\alpha$ synthesis.

Notes: Cells ( $2 \times 10^{6} /$ well in 6-well culture plates) were stimulated with the same volume of PBS (control group), LPS (I $\left.\mu \mathrm{g} / \mathrm{mL}^{2}\right), \mathrm{LPS}$ and Dex (I0-4-I0-7 M), respectively, for 6, 24, and 48 hours. Expression of TNF- $\alpha$ in the culture medium was determined using ELISA assay. Data were expressed as mean \pm SD ( $=3$ ). $* P<0.05$ versus LPS. \#P<0.05 versus $\mathrm{PBS}$.

Abbreviations: h, hours; PBS, phosphate-buffered saline; LPS, lipopolysaccharide; Dex, dexamethasone; TNF, tumor necrosis factor; SD, standard deviation; ELISA, enzymelinked immunosorbent assay.

immunoregulation, and wound healing. ${ }^{28}$ TGF- $\beta$ signaling plays vital roles in mediating pulmonary fibrosis when excessive repair appears. ${ }^{29}$ Wang et al reported that multiwall carbon nanotubes could promote pulmonary fibrosis through TGF- $\beta /$ Smad signaling pathway. ${ }^{30}$ Our data showed that after being stimulated by a relatively higher dose of Dex $\left(10^{-4} \mathrm{M}\right)$ for 48 hours, TGF- $\beta$ was significantly higher compared with that in other groups.

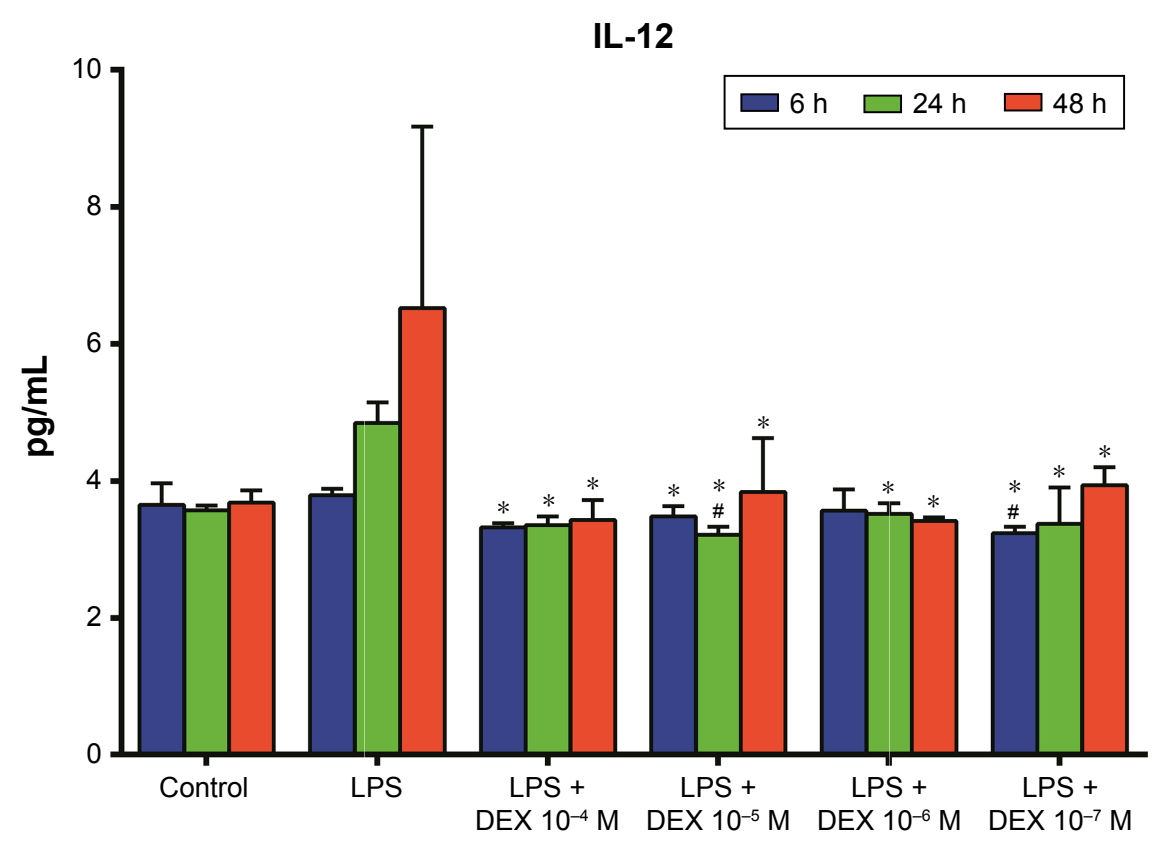

Figure 6 Effect of Dex on IL-12 synthesis.

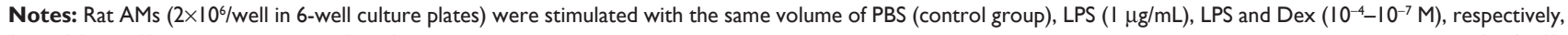
for 6, 24, and 48 hours. Production of IL- 12 in the culture medium was determined using an enzyme-linked immunosorbent assay. Data were expressed as mean \pm SD ( $n=3$ ). $* P<0.05$ versus LPS. ${ }^{*} P<0.05$ versus PBS.

Abbreviations: h, hours; AM, alveolar macrophage; PBS, phosphate-buffered saline; LPS, lipopolysaccharide; Dex, dexamethasone; IL, interleukin; SD, standard deviation. 


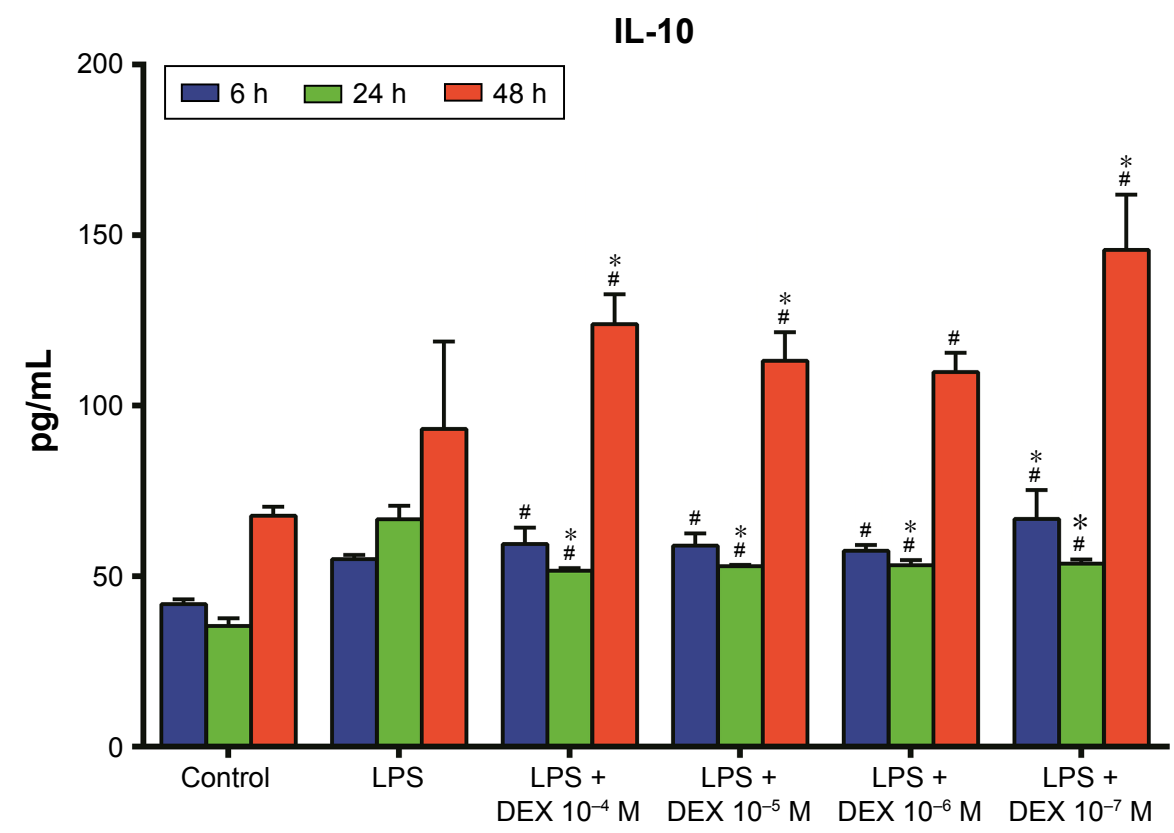

Figure 7 Effect of Dex on IL-10 synthesis.

Notes: Rat AMs ( $2 \times 10^{6} /$ well in 6-well culture plates) were stimulated with the same volume of PBS (control group), LPS (I $\left.\mu \mathrm{g} / \mathrm{mL}\right), \mathrm{LPS}$ and Dex (I0-4 $\left.-10^{-7} \mathrm{M}\right)$, respectively, for 6, 24, and 48 hours. Production of IL- 10 in the culture medium was determined using an enzyme-linked immunosorbent assay. Data were expressed as mean \pm SD ( $=3$ ). $* P<0.05$ versus LPS. ${ }^{*} P<0.05$ versus PBS.

Abbreviations: h, hours; AM, alveolar macrophage; PBS, phosphate-buffered saline; LPS, lipopolysaccharide; Dex, dexamethasone; IL, interleukin; SD, standard deviation.

This might be associated with the pulmonary fibrosis caused by GCs. Further studies are needed to investigate the role of TGF- $\beta$ in ALI/ARDS and the relationship between GCs and TGF- $\beta$.
In summary, our study indicated that high-dose Dex induced the apoptosis of LPS-stimulated rat AMs. The decreased AMs may weaken the self-repair ability of lung tissue and increase the content of TGF- $\beta$ to induce pulmonary fibrosis.

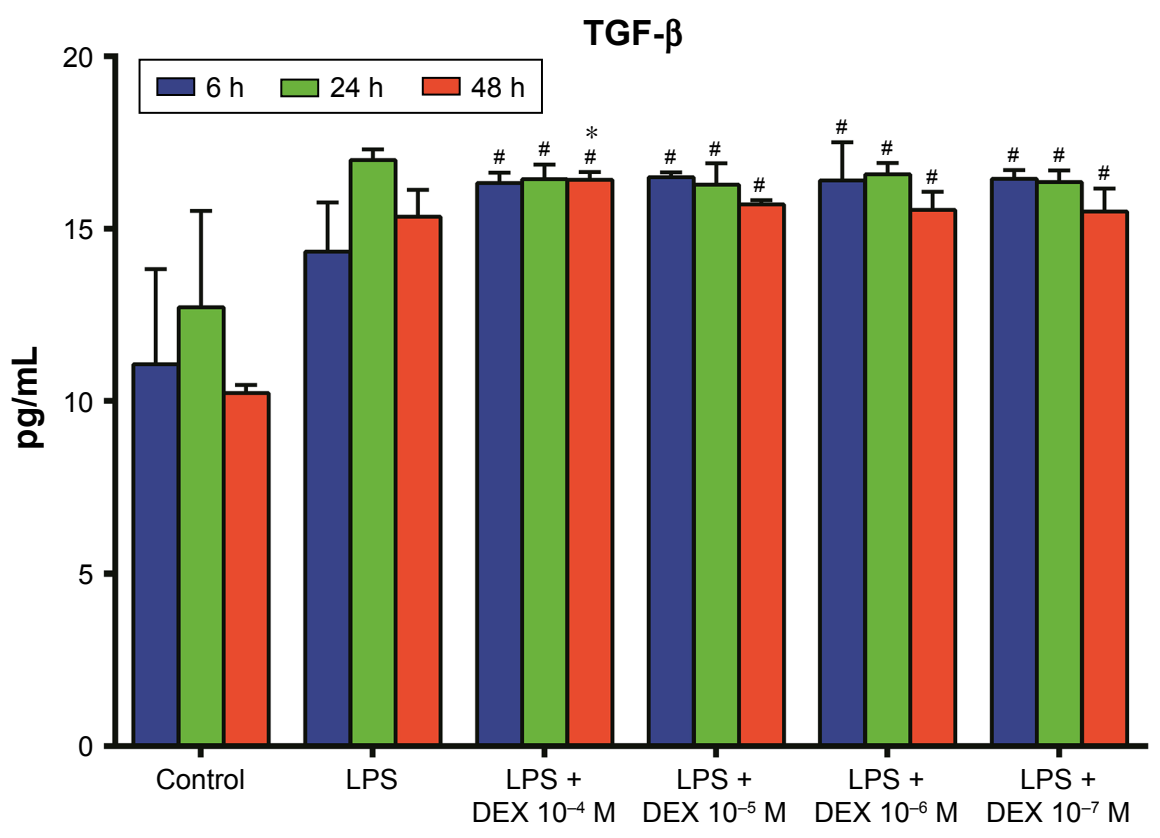

Figure 8 Effect of Dex on TGF- $\beta$ synthesis.

Notes: Rat AMs $\left(2 \times 10^{6} /\right.$ well in 6-well culture plates) were stimulated with the same volume of PBS (control group), LPS (I $\left.\mu \mathrm{g} / \mathrm{mL}\right), \mathrm{LPS}$ and Dex (I0-4 $\left.-10^{-7} \mathrm{M}\right)$, respectively, for 6,24 , and 48 hours. Production of TGF- $\beta$ in the culture medium was determined using an enzyme-linked immunosorbent assay. Data were expressed as mean \pm SD $(\mathrm{n}=3)$. ${ }^{*} \mathrm{P}<0.05$ versus LPS. ${ }^{*} \mathrm{P}<0.05$ versus $\mathrm{PBS}$.

Abbreviations: h, hours; AM, alveolar macrophage; PBS, phosphate-buffered saline; LPS, lipopolysaccharide; Dex, dexamethasone; SD, standard deviation; TGF, transforming growth factor. 


\section{Acknowledgments}

This work was supported by the Sichuan Provincial Health Department (fund 100458). Dr Si Zeng and Dr Hui Qiao were co-first authors for this paper.

\section{Disclosure}

The authors report no conflicts of interest in this work.

\section{References}

1. Zambon M, Vincent JL. Mortality rates for patients with acute lung injury/ARDS have decreased over time. Chest. 2008;133:1120-1127.

2. Bernard GR, Luce JM, Sprung CL, et al. High-dose corticosteroids in patients with the adult respiratory distress syndrome. NEngl J Med. 1987; 317:1565-1570.

3. Meduri GU, Headley AS, Golden E, et al. Effect of prolonged methylprednisolone therapy in unresolving acute respiratory distress syndrome: a randomized controlled trial. JAMA. 1998;280:159-165.

4. Steinberg KP, Hudson LD, Goodman RB, et al. Efficacy and safety of corticosteroids for persistent acute respiratory distress syndrome. N Engl J Med. 2006;354:1671-1684.

5. Lohmann-Matthes ML, Steinmüller C, Franke-Ullmann G. Pulmonary macrophages. Eur Respir J. 1994;7:1678-1689.

6. Dong H, Li J, Lv Y, et al. Comparative analysis of the alveolar macrophage proteome in ALI/ARDS patients between the exudative phase and recovery phase. BMC Immunol. 2013;14:25.

7. Teng D, Pang QF, Yan WJ, Zhao Xin W, Xu CY. The harmful effect of prolonged high-dose methylprednisolone in acute lung injury. Int Immunopharmacol. 2013;15:223-226.

8. Mokart D, Guery BP, Bouabdallah R, et al. Deactivation of alveolar macrophages in septic neutropenic ARDS. Chest. 2003;124:644-652.

9. Nguyen KB, McCombe PA, Pender MP. Increased apoptosis of T lymphocytes and macrophages in the central and peripheral nervous systems of Lewis rats with experimental autoimmune encephalomyelitis treated with dexamethasone. J Neuropathol Exp Neurol. 1997;56:58-69.

10. Joh EH, Gu W, Kim DH. Echinocystic acid ameliorates lung inflammation in mice and alveolar macrophages by inhibiting the binding of

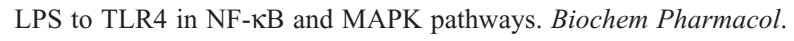
2012;84:331-340.

11. Sprung CL, Caralis PV, Marcial EH, et al. The effects of high-dose corticosteroids in patients with septic shock. A prospective, controlled study. N Engl J Med. 1984;311:1137-1143.

12. Cronin L, Cook DJ, Carlet J, et al. Corticosteroid treatment for sepsis: a critical appraisal and meta-analysis of the literature. Crit Care Med. 1995;23:1430-1439.

13. Behar SM, Martin CJ, Booty MG, et al. Apoptosis is an innate defense function of macrophages against Mycobacterium tuberculosis. Mucosal Immunol. 2011;4:279-287.

14. Castro R, Zou J, Secombes CJ, Martin SA. Cortisol modulates the induction of inflammatory gene expression in a rainbow trout macrophage cell line. Fish Shellfish Immunol. 2011;30:215-223.
15. Wyllie AH. Glucocorticoid-induced thymocyte apoptosis is associated with endogenous endonuclease activation. Nature. 1980;284: $555-556$.

16. Meagher LC, Cousin JM, Seckl JR, Haslett C. Opposing effects of glucocorticoids on the rate of apoptosis in neutrophilic and eosinophilic granulocytes. J Immunol. 1996;156:4422-4428.

17. Hirano S, Asano K, Namba M, Kanai K, Hisamitsu T, Suzaki H. Induction of apoptosis in nasal polyp fibroblasts by glucocorticoids in vitro. Acta Otolaryngol. 2003;123:1075-1079.

18. Haim YO, Unger ND, Souroujon MC, Mittelman M, Neumann D. Resistance of LPS-activated bone marrow derived macrophages to apoptosis mediated by dexamethasone. Sci Rep. 2014;4:4323.

19. Li XJ, Liu DP, Chen HL, Pan XH, Kong QY, Pang QF. Lactoferrin protects against lipopolysaccharide-induced acute lung injury in mice. Int Immunopharmacol. 2012;12:460-464.

20. Maxey TS, Enelow RI, Gaston B, Kron IL, Laubach VE, Doctor A. Tumor necrosis factor-alpha from resident lung cells is a key initiating factor in pulmonary ischemia-reperfusion injury. J Thorac Cardiovasc Surg. 2004;127:541-547.

21. Meduri GU, Muthiah MP, Carratu P, Eltorky M, Chrousos GP. Nuclear factor-kappaB and glucocorticoid receptor alpha-mediated mechanisms in the regulation of systemic and pulmonary inflammation during sepsis and acute respiratory distress syndrome. Evidence for inflammation-induced target tissue resistance to glucocorticoids. Neuroimmunomodulation. 2005;12:321-338.

22. Durrant DM, Metzger DW. IL-12 can alleviate Th17-mediated allergic lung inflammation through induction of pulmonary IL-10 expression. Mucosal Immunol. 2010;3:301-311.

23. Kim HS, Chung DH. TLR4-mediated IL-12 production enhances IFN- $\gamma$ and IL- $1 \beta$ production, which inhibits TGF- $\beta$ production and promotes antibody-induced joint inflammation. Arthritis Res Ther. 2012; 14:R210.

24. Sabat R, Grütz G, Warszawska K, et al. Biology of interleukin-10. Cytokine Growth Factor Rev. 2010;21:331-344.

25. Aggarwal NR, Tsushima K, Eto Y, et al. Immunological priming requires regulatory $\mathrm{T}$ cells and IL-10-producing macrophages to accelerate resolution from severe lung inflammation. J Immunol. 2014;192: 4453-4464.

26. Xia CQ, Peng R, Beato F, Clare-Salzler MJ. Dexamethasone induces IL-10-producing monocyte-derived dendritic cells with durable immaturity. Scand J Immunol. 2005;62:45-54.

27. Visser J, van Boxel-Dezaire A, Methorst D, Brunt T, de Kloet ER, Nagelkerken L. Differential regulation of interleukin-10 (IL-10) and IL-12 by glucocorticoids in vitro. Blood. 1998;91:4255-4264.

28. Skeen VR, Paterson I, Paraskeva C, Williams AC. TGF- $\beta 1$ signalling, connecting aberrant inflammation and colorectal tumorigenesis. Curr Pharm Des. 2012;18:3874-3888.

29. Cong X, Hubmayr RD, Li C, Zhao X. Plasma membrane wounding and repair in pulmonary diseases. Am J Physiol Lung Cell Mol Physiol. 2017;312:L371-L391.

30. Wang $\mathrm{P}$, Nie X, Wang Y, et al. Multiwall carbon nanotubes mediate macrophage activation and promote pulmonary fibrosis through TGF- $\beta$ / Smad signaling pathway. Small. 2013;9:3799-3811.
Drug Design, Development and Therapy

\section{Publish your work in this journal}

Drug Design, Development and Therapy is an international, peerreviewed open-access journal that spans the spectrum of drug design and development through to clinical applications. Clinical outcomes, patient safety, and programs for the development and effective, safe, and sustained use of medicines are the features of the journal, which

\section{Dovepress}

has also been accepted for indexing on PubMed Central. The manuscript management system is completely online and includes a very quick and fair peer-review system, which is all easy to use. Visit http://www.dovepress.com/testimonials.php to read real quotes from published authors. 